Musées, Patrimoine et Culture scientifiques et techniques

$131 \mid 2010$

septembre - octobre 2010

\title{
Le Centre national de la Culture industrielle et du Travail du Luxembourg
}

The National Centre of Industrial Culture and Work of Luxembourg

\section{Antoinette Lorang}

\section{OpenEdition}

Journals

Édition électronique

URL : http://journals.openedition.org/ocim/109

DOl : 10.4000/ocim.109

ISSN : 2108-646X

Éditeur

OCIM

Édition imprimée

Date de publication : 1 septembre 2010

Pagination : $19-26$

ISSN : 0994-1908

\section{Référence électronique}

Antoinette Lorang, « Le Centre national de la Culture industrielle et du Travail du Luxembourg », La Lettre de l'OCIM [En ligne], 131 | 2010, mis en ligne le 01 septembre 2012, consulté le 14 novembre 2019. URL : http://journals.openedition.org/ocim/109; DOI : 10.4000/ocim.109 


\section{Le Centre national de la Culture industrielle et du Travail de Luxembourg}

Antoinette Lorang *

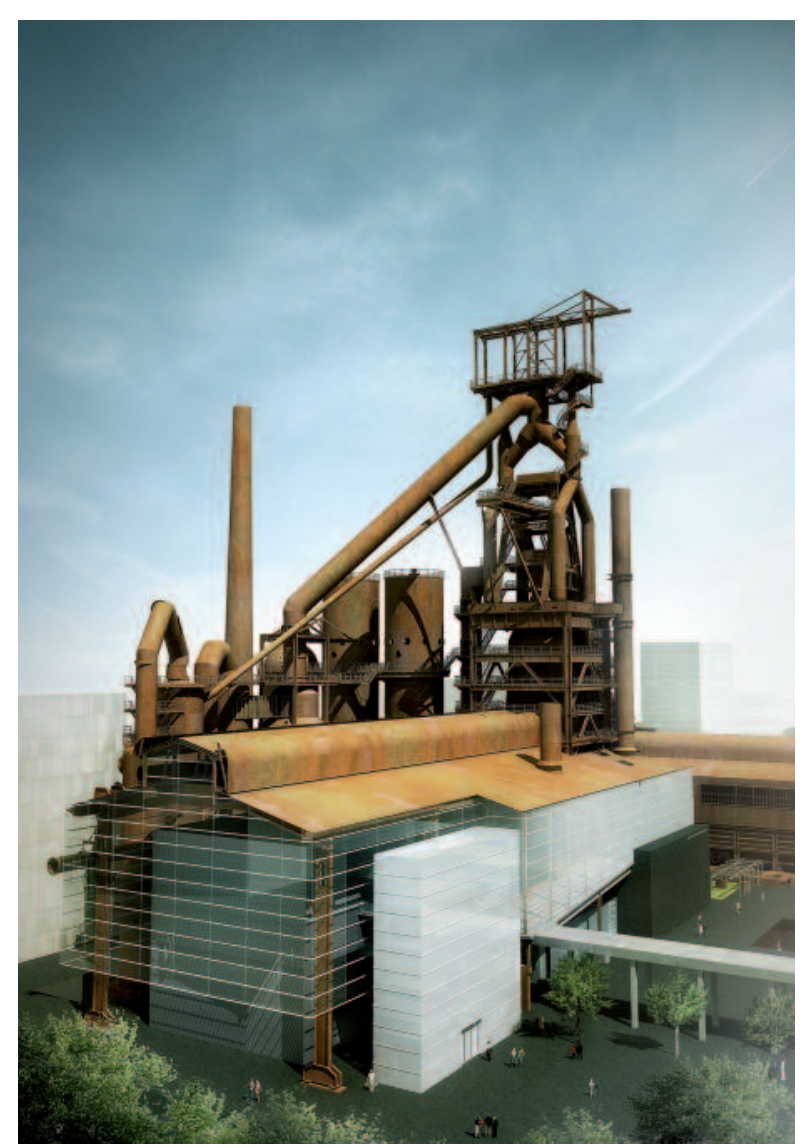

Le haut fourneau de Belval reconverti en Centre national de la Culture industrielle et du Travail. Le projet du bureau $d^{\prime}$ 'architectes luxembourgeois Beyler et François prévoit l'intégration d'un nouveau bâtiment cube sous l'enveloppe de la halle des coulées. (c) Le Fonds Belval

* Antoinette Lorang est historienne de l'art, chargée de mission Culture et Communication auprès du Fonds Belval, établissement public chargé de la construction de la Cité des Sciences antoinette.lorang@fonds-belval.lu
Intégré à la Cité des Sciences, de la Recherche et de I'Innovation, le projet urbain de reconversion de la friche industrielle de Belval à Esch-sur-Alzette au Luxembourg, le Centre national de la Culture industrielle et du Travail est conçu comme un lieu de mémoire du passé sidérurgique de la région mais également comme l'élément dynamisant et animateur du réseau national de culture scientifique, technique et industrielle.

Deux hauts fourneaux implantés à Belval au sudouest du Grand-Duché de Luxembourg ont survécu, au moins partiellement, aux différentes crises de la sidérurgie. S'ils ne verront plus aucune coulée réelle de fonte, un nouveau rôle leur est attribué dans le contexte de la Cité des Sciences, de la Recherche et de l'Innovation, un grand projet de l'État luxembourgeois en voie de création sur l'ancienne friche industrielle de Belval.

Selon le concept de conservation retenu par le gouvernement, un des deux hauts fourneaux est conservé pour accueillir le Centre national de la Culture industrielle et du Travail, le deuxième a été démantelé jusqu’à la silhouette. Ensemble, les vestiges industriels représentent un landmark impressionnant dans le paysage. Une partie des espaces libérés par le démantèlement seront occupés par des nouveaux immeubles réalisés dans l'intérêt de l'université du Luxembourg qui sera le principal utilisateur du quartier de la Terrasse des Hauts Fourneaux. Si les travaux sur le site de Belval vont bon train, les adeptes du projet pour le Centre national de la Culture industrielle et du Travail doivent encore patienter quelque peu. Dans une première phase, 
débutant à la fin de l'année 2010, seront réalisés les travaux de restauration des hauts fourneaux.

\section{Quelques mots sur la sidérurgie au Luxembourg}

Pour bien comprendre l'enjeu de conservation des hauts fourneaux de Belval, il faut se rendre compte de l'importance de la sidérurgie pour le développement économique du Grand-Duché. En effet, l'histoire des deux derniers siècles du Luxembourg, et tout particulièrement celle du bassin minier, est indissociable de la sidérurgie. Le Luxembourg compte même parmi les premiers producteurs mondiaux d'acier dès le début du XXe siècle. Jusqu'aux années 1970, l'industrie du fer reste le secteur le plus important de l'économie luxembourgeoise. Mais, avec la crise de l'acier, l'industrie lourde cesse de jouer un rôle prépondérant, tandis que le secteur tertiaire, surtout le marché des finances, se développe au Luxembourg grâce à une politique de diversification du gouvernement.

L'essor de la sidérurgie luxembourgeoise depuis la seconde moitié du XIXe siècle est dû aux ressources naturelles de minerai de fer ( "Minett») dans la partie sud du Grand-Duché, le «bassin minier », qui est un prolongement des gisements plus étendus en Lorraine. De 1850 à 1870, une grande partie du minerai extrait au Luxembourg est destiné à l'exportation. Avec la loi de 1870 sur les concessions minières, les pouvoirs publics prennent soin de lier les intérêts des maîtres de forges luxembourgeois à ceux de l'État : des nouvelles concessions minières ne sont en général plus attribuées que sous condition que le minerai soit transformé dans le pays, favorisant ainsi la création d'usines à proximité des minières.

Les premiers hauts fourneaux du bassin minier luxembourgeois sont construits de 1871 à 1873 à Esch-sur-Alzette, centre administratif de la région sud du Luxembourg qui connaît un développement considérable suite à l'industrialisation, sa population passant de 2200 en 1870 à 13000 habitants en 1906 quand elle est élevée au rang d'une ville, pour atteindre 21000 habitants en 1914.

Dans les années 1890, la sidérurgie luxembourgeoise entre dans une phase déterminante. De nouvelles usines intégrant agglomérations, hauts fourneaux, aciéries et laminoirs sont construites parmi lesquelles Esch-Belval, installée de 1909 à 1912 par la société allemande « Gelsenkirchener Bergwerks AG », une des usines les plus modernes d'Europe est créée dans le bassin minier luxembourgeois. Elle compte six hauts fourneaux.
À l'aube de la Première Guerre mondiale, le GrandDuché de Luxembourg se positionne au $6^{\mathrm{e}}$ rang des producteurs sidérurgiques du monde. La création de l'Arbed (Aciéries Réunies de Burbach Eich Dudelange) en 1911, marque un point décisif pour le développement futur de la sidérurgie luxembourgeoise. L'Arbed existe jusqu'en 2002. En fusionnant avec Aceralia en Espagne et Usinor/Sacilor en France, elle devient Arcelor et premier producteur mondial au début du nouveau millénaire, rachetée en 2006 par Mittal Steel pour devenir ArcelorMittal.

De 1965 à 1979 l'usine subit de grandes transformations. Les anciens hauts fourneaux sont démolis et remplacés par les grands hauts fourneaux modernes $\mathrm{A}, \mathrm{B}, \mathrm{C}$ qui somme toute, n'auront qu'une vie courte. La crise dans la sidérurgie à partir des années 1970, déclenche la fermeture au fur et à mesure des anciens sites industriels du bassin minier. En 1981, la dernière mine de fer du pays ferme. Désormais, la totalité du minerai est importée. En 1997, le dernier haut fourneau s'éteint à Esch-Belval, l'usine est passée à la filière électrique.

\section{Prise de conscience du patrimoine industriel}

Au Luxembourg, la prise de conscience du patrimoine industriel remonte à l'époque de la grande crise de la sidérurgie. C'est la perspective de la perte qui fait naître le besoin de conserver et de restaurer, surtout à une époque où les changements se font de plus en plus rapides. La première initiative en ce sens a été la création d'un musée des Mines en 1973, suite à la fermeture de la dernière mine à Rumelange. Sur une échelle plus large, c'est la politique du ministère de la Culture qui, à l'époque, pose

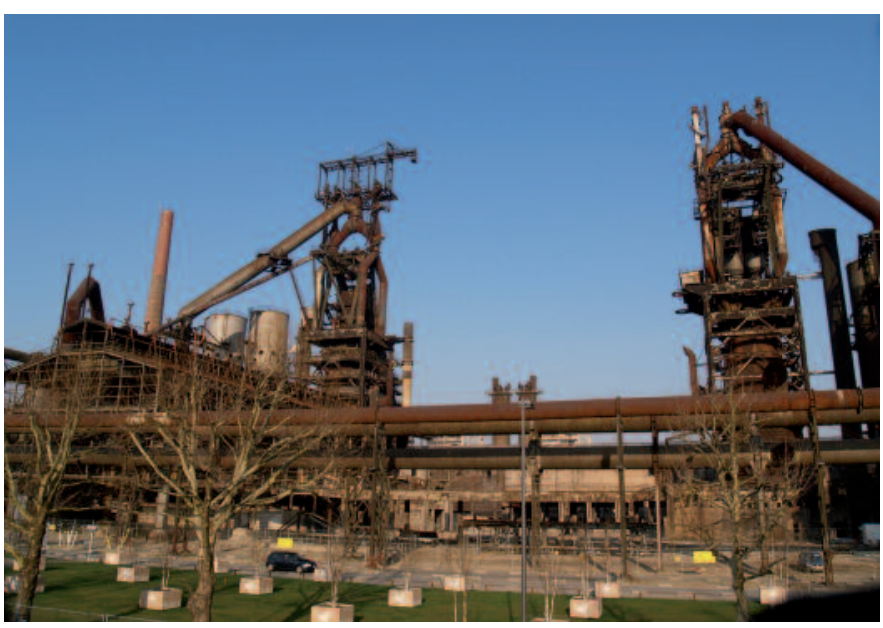

Les deux hauts fourneaux de Belval : état actuel (c) OCIM 
les jalons pour la valorisation du patrimoine industriel. La loi de 1927 sur la protection des sites et monuments est remplacée en 1983 par une nouvelle loi introduisant la notion de patrimoine industriel. En 1986 le service des Sites et Monuments nationaux nouvellement créé entame la réalisation d'un Parc industriel et ferroviaire au Fond-de-Gras près de Differdange. Parallèlement aux initiatives gouvernementales, un mouvement spontané populaire commémore les souvenirs d'un passé brusquement arrêté à travers la création de musées et la mise en place de monuments : les associations des anciens mineurs, les collectionneurs d'objets et de documents, les passionnés de l'histoire locale.

De nombreux musées documentant l'histoire industrielle ont vu le jour depuis lors. Néanmoins beaucoup de vestiges ont aussi été détruits ou laissés à l'abandon. Certaines localités devenues propriétaires de friches industrielles ont fait table rase des installations techniques dès leur désaffectation pour valoriser les terrains ou simplement pour faire " propre ». Souvent le patrimoine est sacrifié cependant parce qu'il se trouve sur des sites industriels encore en activité sujets à des changements fréquents dus à l'évolution technologique et économique.

Pour le site de Belval-Ouest on constate une approche particulière qui peut être interprétée comme le fruit du travail de sensibilisation pour le patrimoine industriel mené au préalable. En 1996, même avant l'arrêt définitif du dernier haut fourneau, la Chambre des Députés s'exprime en faveur de la conservation d'un des deux hauts fourneaux. En 1998, elle propose de racheter le deuxième haut fourneau et d'étudier la possibilité d'en faire un musée. Une association de défense du patrimoine, la Fondation Bassin Minier, est chargée d'établir un concept pour ce musée. En 2000, les hauts fourneaux sont inscrits à l'Inventaire supplémentaire des Sites et Monuments nationaux en vue de leur protection.

Cet engagement pour la conservation du patrimoine industriel est lié en partie au fait qu'il s'agit des derniers hauts fourneaux du Luxembourg, mais aussi au fait que leur valeur commerciale est restreinte. Si le groupe Arbed réussit à vendre le troisième haut fourneau de Belval datant de 1979 à une société chinoise, les deux autres n'avaient manifestement plus d'intérêt pour la production. L'Arbed se décide à léguer le haut fourneau $\mathrm{A}$ au peuple luxembourgeois et l'État rachète le deuxième haut fourneau dans le contexte des acquisitions de terrains de la friche de Belval.

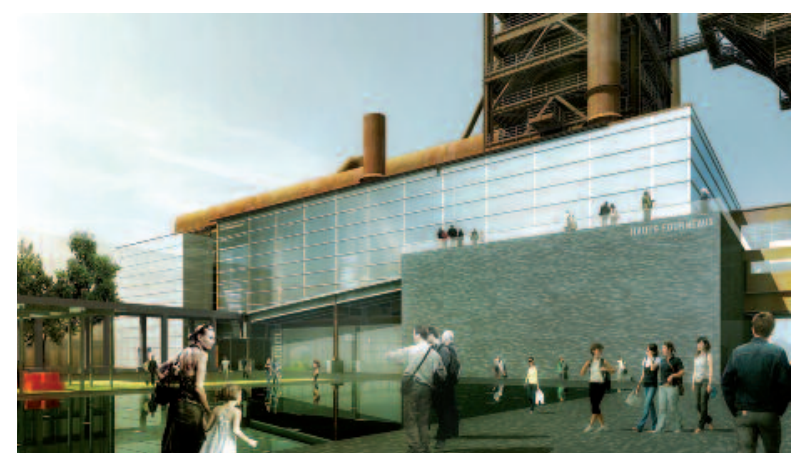

Projet d'entrée du Centre national de la Culture industrielle et la halle des coulées recouverte d'une nouvelle enveloppe vitrée. Entre les deux hauts fourneaux sera aménagée une place publique suivant un concept du paysagiste français Michel Desvigne. (c) Le Fonds Belval

\section{Les hauts fourneaux de Belval dans le contexte de la Cité des Sciences}

Les hauts fourneaux font aujourd'hui partie du projet de reconversion de la friche industrielle de Belval en quartier urbain. Sur une surface de quelques 15 ha sur un total de 120 ha, le gouvernement luxembourgeois a entamé la réalisation d'une "Cité des Sciences, de la Recherche et de l'Innovation » qui consiste en un ensemble d'infrastructures pour l'université du Luxembourg et les centres de recherche publics ainsi que pour des activités économiques, socioculturelles et sportives. Le concept de conservation des vestiges industriels retenu par le gouvernement en 2005 part sur l'idée d'un " monument dans la cité » faisant partie de la vie urbaine. Un des deux hauts fourneaux est conservé avec sa halle des coulées et ses principaux équipements. Le deuxième haut fourneau est réduit jusquà la silhouette, la halle des coulées est démolie, le haut fourneau est mis à nu dans sa tour carrée et apparaît comme une sculpture surdimensionnée. La plupart des bâtiments communs aux deux hauts fourneaux sont également démontés tandis que le bâtiment de la charge de minerai, la « Moellerei », est partiellement conservée avec ses installations techniques et partiellement réaffectée comme bibliothèque universitaire. Les surfaces libérées sont aménagées comme espace public ou reconstruites avec des bâtiments contemporains répondant aux besoins de l'université et des centres de recherche.

La Cité des Sciences, de la Recherche et de l'Innovation est le projet phare de la reconversion des friches industrielles dans le bassin minier luxembourgeois. Avec ce projet, l'État entend relancer les investissements, tant publics que privés et la 
renaissance des activités socio-économiques dans le sud du Grand-Duché de Luxembourg qui a ressenti plus particulièrement les restructurations du secteur métallurgique. La Cité des Sciences s'articule autour : - de l'enseignement supérieur, de la recherche et de l'enseignement secondaire ;

- de la vie estudiantine ;

- des activités de start-up et d'entreprises de la nouvelle économie ;

- des services et administrations de l'État ;

- de la vie culturelle, des sports et des loisirs.

La Cité des Sciences accueillera deux facultés de l'université du Luxembourg, la faculté des Sciences, de la Technologie et de la Communication et la faculté des Lettres, des Sciences Humaines, des Arts et des Sciences de l'Éducation et le département Économie de la faculté de Droit, d'Économie et de Finance ainsi que plusieurs centres de recherche publics.

La Cité des Sciences qui vient investir la Terrasse des Hauts Fourneaux, fera partie d'un quartier urbain multifonctionnel qui se développe autour des vestiges industriels conservés.

\section{Le concept du Centre national de la Culture industrielle et du Travail}

La nouvelle destination des hauts fourneaux dans le contexte de la Cité des Sciences a conditionné l'orientation de leur exploitation en tant que Centre national de la Culture industrielle et du Travail. Les premières réflexions au sein d'un groupe interdisciplinaire chargé d'étudier le concept et quelques sondages auprès d'un certain nombre de groupes cibles ont été unanimes sur l'option de créer un lieu de documentation de la sidérurgie pour maintenir la mémoire du passé et en même temps un espace d'activités temporaires orientées sur des thèmes d'actualité en matière de technologies, de sciences et de travail. Conformément à cette approche, le concept développé pour le Centre national de la Culture industrielle et du Travail prévoit plusieurs volets :

- le centre de découverte de la sidérurgie,

- le centre culturel de la Cité des Sciences,

- les activités pédagogiques et de formations,

- les réseaux de la culture industrielle, scientifique et technique.

\section{Un centre de découverte de la sidérurgie}

Le centre de découverte de la sidérurgie se composera en partie d'activités permanentes sous forme d'un parcours de visite du haut fourneau et en partie d'activités temporaires sous forme d'expositions et d'autres manifestations.

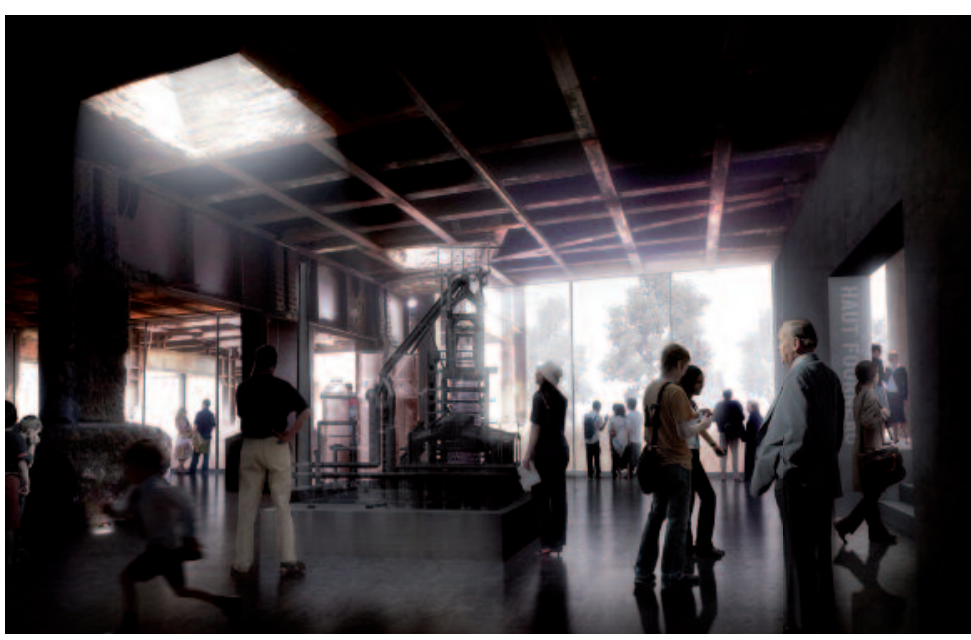

L'espace où circulaient jadis les poches à fonte sera transformé en hall $d$ 'accueil servant également à des manifestations sur la culture industrielle, le travail, les sciences et les technologies qui détermineront la vie de demain.

(C) Le Fonds Belval

Le Centre national de la Culture industrielle et du Travail se vouera à l'histoire des hauts fourneaux de Belval depuis leur première mise à feu en 1911 jusqu'à la dernière coulée en 1997. Le long du parcours, les visiteurs découvriront les principaux éléments du processus de la fonte, à commencer par le bâtiment de la charge de minerai ou « Moellerei » en passant par le haut fourneau jusqu'à la halle des coulées où la fonte liquide se déversait vers les poches à fonte. La montée vers la plateforme du gueulard à quelques $40 \mathrm{~m}$ de hauteur, l'accès à l'intérieur du haut fourneau et une coulée virtuelle seront les « highlights » du parcours. Tout au long du circuit, des bornes interactives diffuseront des informations sur le fonctionnement des installations industrielles, les sidérurgistes, ouvriers, ingénieurs, techniciens, l'évolution des conditions de travail et les enjeux du site industriel.

Des expositions temporaires et d'autres activités sur l'histoire de la sidérurgie complèteront les informations diffusées sur le circuit de visite. Pour alimenter ces activités, le Centre de la Culture industrielle et du Travail lancera des recherches et soutiendra des études historiques sur le passé industriel et les répercussions sur la société, l'économie, la vie culturelle...

\section{Un centre culturel de la Cité des Sciences}

Enraciné dans le passé avec ses structures impressionnantes de l'industrie lourde, le Centre national de la Culture industrielle et du Travail aura aussi une vocation de lieu culturel de la Cité des Sciences ouvert sur des thèmes de la recherche scientifique et technologique contemporaine dans le but de diffuser 
des informations et des connaissances au grand public. Le Centre est ainsi destiné à stimuler les réflexions sur l'évolution des technologies et leur impact sur notre vie quotidienne et le travail, les mutations de la société et des mentalités ou encore le développement architectural, urbain et environnemental lié à l'évolution des techniques et des matériaux. Face aux crises économiques et écologiques avec leurs conséquences néfastes au niveau de l'emploi et de la qualité de vie, il s'avère de plus en plus important de montrer comment sont abordés les problèmes qui se posent et quelles pourraient être des solutions pour l'avenir. À travers des expositions, conférences, colloques, festivals, le Centre national de la Culture industrielle et du Travail sensibilisera le public pour les thèmes déterminant la vie de demain. Le Centre offrira aussi une plateforme intéressante aux institutions universitaires et de recherche pour présenter au public leurs activités scientifiques et des projets d'étudiants.

\section{Les activités pédagogiques et de formations}

Le Centre national de la Culture industrielle et du Travail s'adressera aux usagers du site universitaire ou du site de Belval aussi bien qu'aux visiteurs occasionnels qui recherchent un lieu de loisirs et de divertissement, soit individuel, en groupe ou en famille. Ainsi son offre d'activités sera très diversifiée. Il aura une importante mission pédagogique d'un côté par la transmission de savoirs et de pratiques de la sidérurgie ancienne aux élèves des classes d'école, de l'autre côté par l'offre d'ateliers, de stages et de visites guidées autour des thèmes évoqués dans les expositions. Le Centre vise par ailleurs un haut degré de participation du public dans la conception des activités.

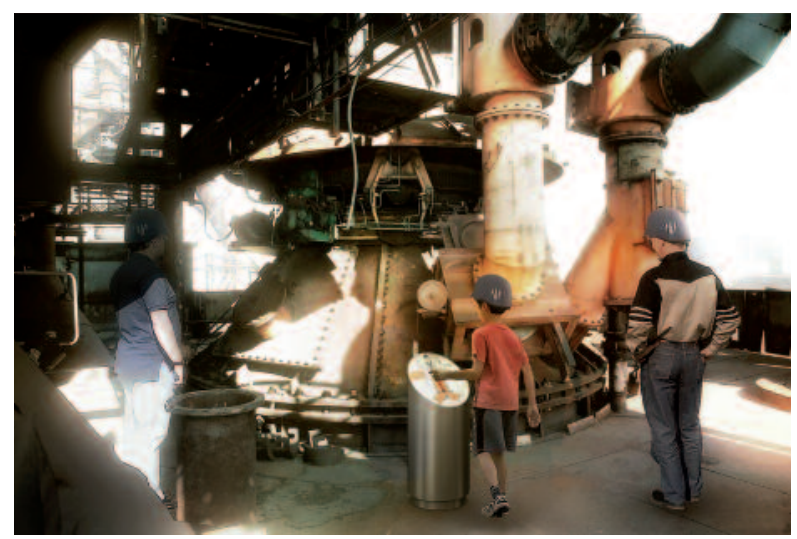

Sur un parcours de visite, le public découvrira les principaux éléments du processus de la fonte. La station gueulard, où se faisait l'alimentation du haut fourneau, est située à une hauteur de $40 \mathrm{~m}$ et sera accessible par ascenseur. (c) Le Fonds Belval
Les réseaux de la culture industrielle, technique et scientifique

Grâce à des initiatives en majeure partie privées soutenues par l'État ou les communes, il existe au Luxembourg bon nombre de musées ou de centres de documentation œuvrant dans l'intérêt de la conservation du patrimoine industriel aussi bien matériel qu'immatériel. Une mission du Centre national de la Culture industrielle et du Travail sera la création d'un réseau de la culture industrielle, technique et scientifique qui réunirait tous les acteurs travaillant sur ces sujets. Le but sera de soutenir les petits musées dans leurs activités et d'augmenter leur visibilité à travers des manifestations sur des thèmes fédérateurs communs, mais aussi de coopérer avec des entreprises et institutions de recherche afin de créer une plateforme d'échange et des liens vers l'actualité du monde économique.

Au niveau de la Grande Région, plusieurs initiatives ont déjà été lancées pour créer des réseaux de la culture industrielle, en Sarre notamment, "Industriekultur Saar », ou transfrontalier comme le réseau de la « Route du fer » qui relie les sites de Völklingen en Sarre, Petite Rosselle en Lorraine et Fond-deGras au Luxembourg et ces sites représentent des maillons dans le réseau européen ERIH. Avec la conservation et la valorisation du haut fourneau d'Uckange en Lorraine et des hauts fourneaux de Belval, le réseau de la Grande Région devient encore plus intéressant. En prenant en compte aussi le site de Neunkirchen, on recèle quatre hauts fourneaux encore en place dans un espace de quelques $12000 \mathrm{~km}^{2}$. Si l'on considère par ailleurs le haut fourneau " couché » de Longwy on distinguera cinq variantes possibles d'approches envers le patrimoine

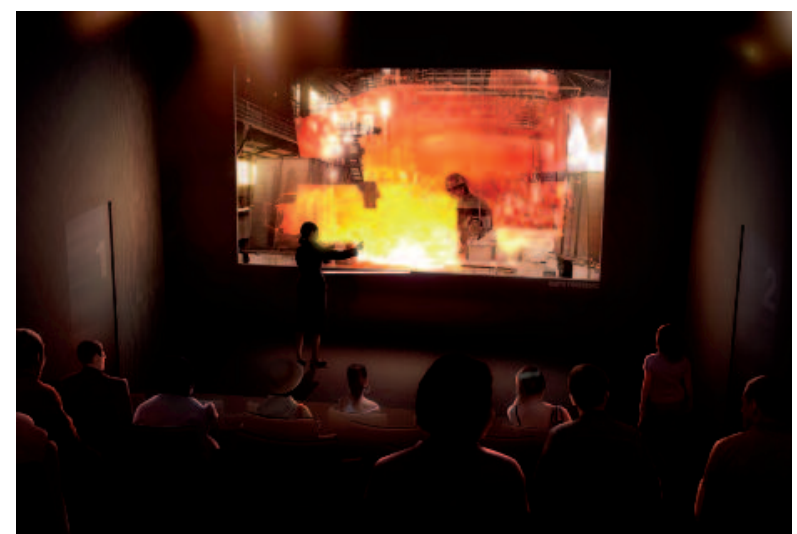

Des espaces pédagogiques incluant une salle de projection seront aménagés dans le nouveau bâtiment cube. Une coulée virtuelle sera présentée lors du parcours de visite dans cette salle donnant sur la halle des coulées et le haut fourneau réel. () Le Fonds Belval 
industriel. Le site de Völklingen « Europäisches Zentrum für Kunst und Industriekultur », classé patrimoine mondial, le plus grand d'entre eux et le plus complet, offre des parcours de visites du monument industriel et de nombreuses activités culturelles, Neunkirchen a conservé ses hauts fourneaux comme "landmark ", un château d'eau a été transformé en cinéma, le haut fourneau d'Uckange est valorisé à travers un projet artistique de mise en lumière et des activités culturelles, à Longwy subsiste seulement le creuset d'un haut fourneau resté en place après la démolition de l'usine. Les hauts fourneaux de Belval, finalement, se trouvent en plein milieu d'un nouveau quartier urbain en devenir, la Cité des Sciences, où ils intégreront la vie quotidienne des étudiants, des chercheurs et du public fréquentant les infrastructures publiques et commerciales.

\section{Le projet architectural}

Le projet de valorisation des hauts fourneaux dans l'intérêt du Centre national de la Culture industrielle et du Travail prévoit l'aménagement de plusieurs espaces d'accueil et d'activités dans l'un des deux hauts fourneaux et la construction d'un nouveau bâtiment sous forme d'un cube dans l'enceinte de la halle des coulées. L'idée du projet porte sur l'exploitation de volumes existants du haut fourneau le mieux conservé en ajoutant des éléments nouveaux nécessaires pour le bon fonctionnement de l'ensemble sans pourtant modifier la structure existante. Les éléments nouveaux se distinguent des parties anciennes par le choix de matériaux et de formes contemporaines mais minimalistes.

Les espaces du Centre national de la Culture industrielle et du Travail se composent de plusieurs éléments :

- le hall central,

- le parcours de visite des hauts fourneaux,

- l'espace d'expositions temporaires,

- le nouveau cube.

\section{Le hall central}

Le hall central se situe en dessous de la halle des coulées, à cheval entre les soubassements du haut fourneau et le nouveau cube. L'entrée principale donne sur un passage couvert situé sur l'axe principal de circulation des piétons sur le site, l'ancien « highway » qui desservait les trois hauts fourneaux. Le grand hall central servira de lieu d'accueil et d'information au public. Ici se trouveront la réception et un café-bar, l'accès aux vestiaires et sanitaires. Le hall sera le point de départ et d'arrivée de la visite du haut fourneau, il donnera accès à l'espace d'expositions temporaires dans les soubassements. Des manifestations accompagnant les expositions sont également prévues dans le grand hall. Par ailleurs, à l'extérieur, du côté sud, sera aménagé un « jardin de la fonte » où sont conservées des traces des voies ferrées et une poche à fonte dans un cadre de végétation pionnière typique des friches industrielles.

\section{Le parcours de visite du haut fourneau}

Le parcours de visite comprendra l'accès au haut fourneau, à la « Moellerei » (bâtiment de la charge de minerai) et à la halle des coulées. Les vestiges industriels ne sont a priori pas conformes pour accueillir le public. Des aménagements et nouveaux équipements sont nécessaires pour garantir la fonctionnalité du centre culturel et pour assurer la sécurité des visiteurs. Le point de départ et d'arrivée des parcours de visite sera dans le hall central. Les planchers du haut fourneau jusqu'au gueulard seront accessibles par un nouvel ascenseur dans la cage existante. Les planchers ne subiront pas de modifications majeures. Des nouveaux escaliers, des passages et des dispositifs en vue de la sécurité des visiteurs seront mis en place tout au long du parcours.

\section{L'espace d'expositions temporaires}

Les soubassements du haut fourneau seront aménagés pour des expositions temporaires. Il s'agit d'un espace spectaculaire où d'importantes traces du processus industriel sont conservées qui seront valorisées à travers une mise en scène adéquate. Des perspectives vers la fondation du haut fourneau s'ouvriront sur l'espace d'exposition.

Le cube : documentation, formation, recherche, pédagogie, administration

Un nouveau volume, le bâtiment cube, est prévu dans l'enceinte de la halle des coulées du haut fourneau. Le bâtiment comprendra cinq niveaux. Le rezde-chaussée est conçu comme une salle multifonctionnelle qui servira de galerie d'exposition et de manifestations temporaires. Cette salle aura un accès direct depuis le passage couvert et fonctionnera en autonomie par rapport au reste du bâtiment. L'accès aux étages supérieurs se fera via le volume extrapolé contenant les escaliers, l'ascenseur et les sanitaires. Le premier étage accueillera une bibliothèque-médiathèque. Aux troisième et quatrième étages se situeront les espaces pédagogiques et une salle de projection. La salle de projection sera également accessible à partir du plancher des coulées du haut fourneau. Le dernier étage du cube sera réservé à l'administration du centre. 


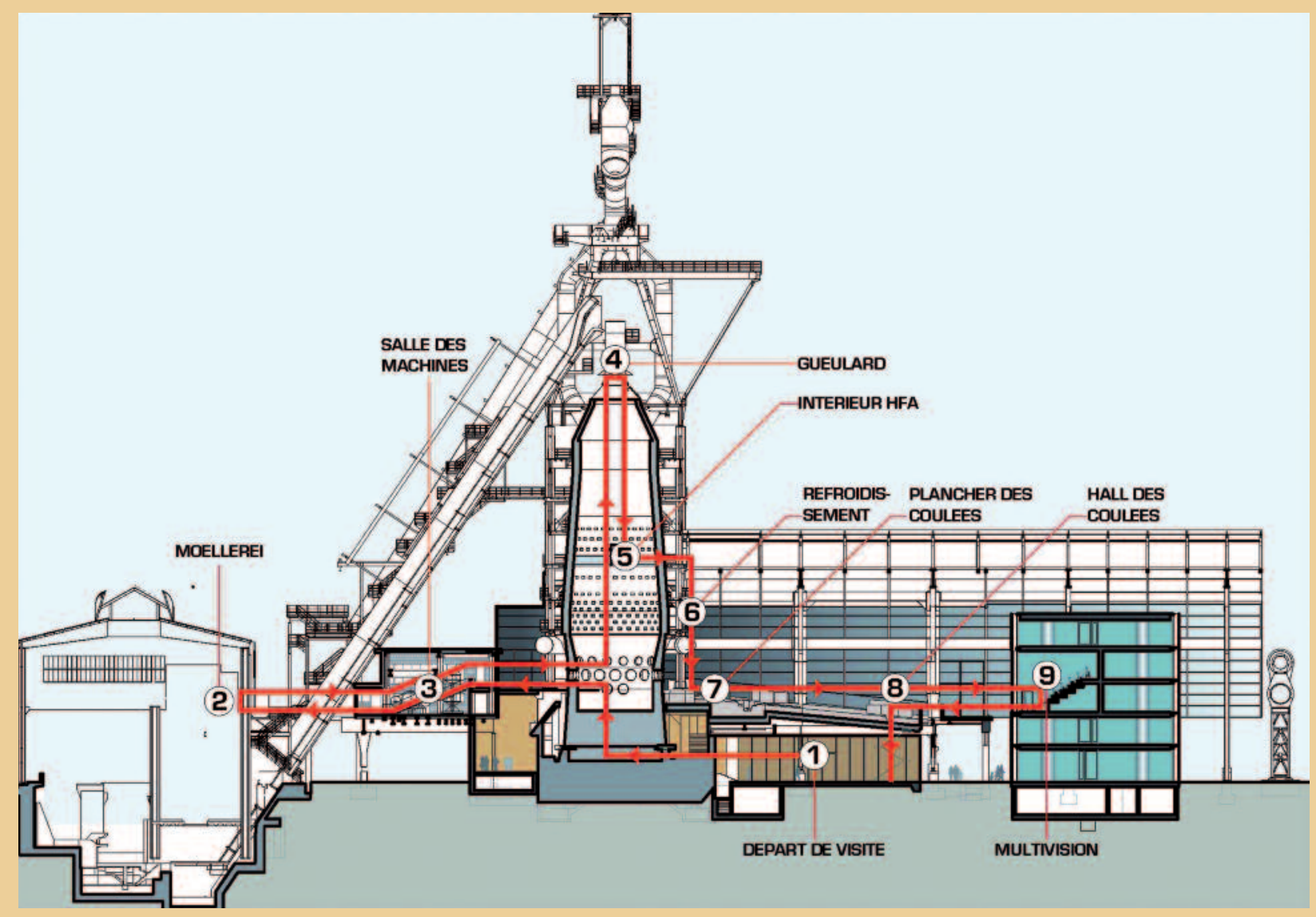

() Le Fonds Belval

Le grand circuit de visite du haut fourneau prend son départ dans le hall central du Centre national de la Culture industrielle et du Travail.

La station 1, au pied de l'ascenseur donne toutes les explications concernant le fonctionnement d'un haut fourneau. Une présentation audiovisuelle, des schémas techniques et une maquette détaillée de l'installation expliquent tous les réseaux fonctionnels de la machine. La station 2 dans la «Moellerei » documente les silos des minerais et de coke ainsi que les équipements respectifs à la préparation de la charge. La station 3 dans la salle des machines du skip montre le treuil qui actionnait les deux bennes d'alimentation du haut fourneau. La station 4 se trouve sur le plancher du gueulard. Cette station est le point culminant du circuit. Située à plus de $40 \mathrm{~m}$, elle offre une vue panoramique sur la Terrasse des Hauts 


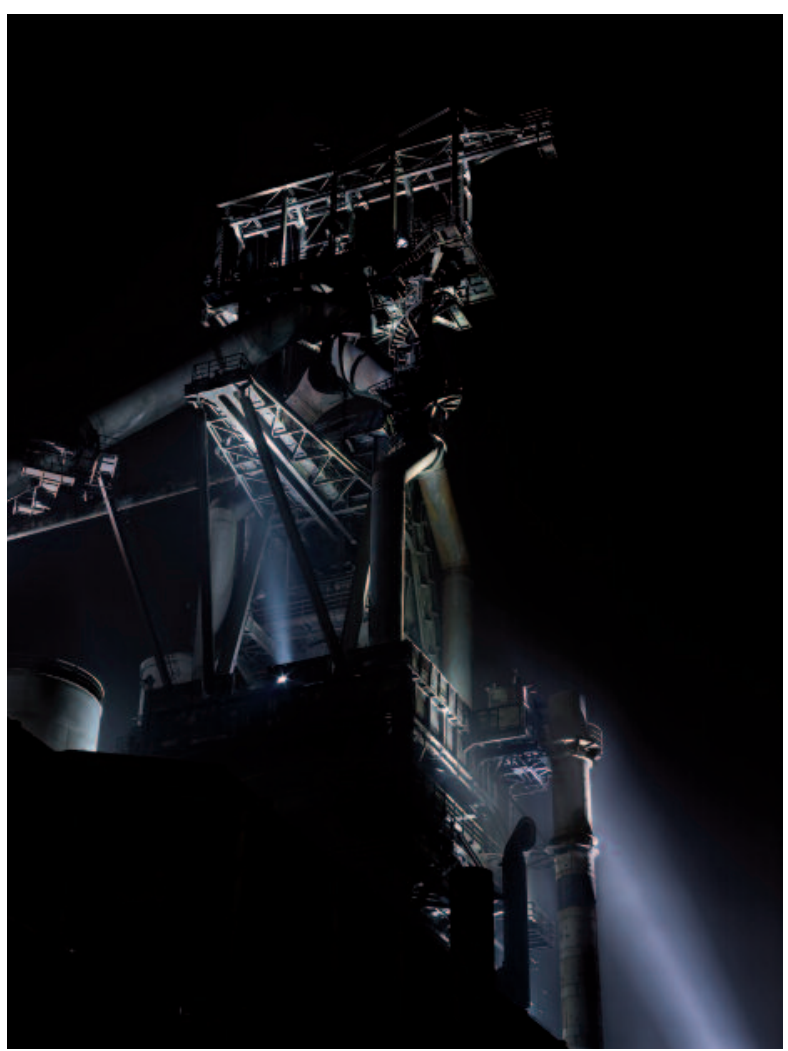

Les hauts fourneaux seront illuminés suivant un concept de l'artiste allemand Ingo Maurer, travaillant la lumière blanche pour redonner aux vestiges leur rayonnement $d$ 'antan. () Tom Vack

\section{L'illumination des hauts fourneaux}

Les hauts fourneaux ont depuis leur origine dominé le site de Belval. Ils ont marqué de jour et de nuit le paysage de l'agglomération d'Esch-sur-Alzette. Dans la nouvelle configuration du site, ils se retrouvent au cœur de la Cité des Sciences, entourés d'activités universitaires, de commerces et de brasseries. Pour leur rendre leur rayonnement d'antan, un concept d'illumination a été élaboré par l'artiste de renommée internationale Ingo Maurer qui soulignera la présence des structures industrielles dans un environnement résolument contemporain. Le projet applique la lumière blanche afin de créer un jeu de contrastes entre ombres et lumières. Un essai de l'éclairage a été fait en octobre 2008 qui a confirmé le concept. La lumière redessine parfaitement les formes de chaque élément et rend le monument visible de loin rappelant l'époque où les hauts fourneaux étaient encore en service.

\section{L'évolution du projet}

Les grands projets d'investissement de l'État luxembourgeois au-dessus d'un certain seuil doivent être autorisés par une loi. Comme le projet de valorisation des hauts fourneaux est un projet exceptionnel qui n'était pas connu dans tous ses éléments au départ, une première loi a été votée par la Chambre des Députés en 2003 pour financer les travaux de mise en sécurité et de démantèlement des hauts fourneaux. Ces travaux étaient achevés fin 2009. Le 8 avril de la même année, le gouvernement a donné son accord de principe pour la réalisation des travaux d'aménagements et de construction pour le Centre national de la Culture industrielle et du Travail et le projet a été avisé favorablement par le Conseil d'État.

Suite à la crise économique, le gouvernement a décidé au début 2010 de scinder la réalisation du Centre national de la Culture industrielle et du Travail en deux phases. Dans la première phase seront menés à bien les travaux de restauration et de traitement de surfaces des hauts fourneaux. Pendant cette période, le concept du Centre national de la Culture industrielle et du Travail sera affiné et le programme sera concrétisé pour être réalisé dans une deuxième phase.

Cet article représente une version élargie d'une conférence tenue dans le cadre des Journées Hubert Curien 2010 à Esch-sur-Alzette, Grand-Duché de Luxembourg

\section{Bibliographie}

Le Fonds Belval (éd.) Concept de conservation des hauts fourneaux de Belval, Les Cahiers du Fonds Belval, Luxembourg, 2006, 147 p.

Le Fonds Belval (éd.) Proposition d'un concept pour le centre national de la culture industrielle, Les Cahiers du Fonds Belval, Luxembourg, 2004, 92 p.

Le Fonds Belval (éd.) Magazine, le périodique du fonds Belval, nº1, 2009, pp. 22-31.

Informations sur la Cité des Sciences et les publications du Fonds Belval : www.fonds-belval.lu

Lorang, A. Le patrimoine industriel au Luxembourg : de la commémoration vers une appropriation événementielle, dans : Guide culturel $d u$ Luxembourg. Luxembourg : Éditions Ilôts, 2007, pp. 262-275.

Fondation Bassin Minier (éd.) Sur les traces du passé. Tourisme industriel au sud du Luxembourg. Luxembourg, 2009, 68 p. 